\title{
La vulnérabilité - un concept complexe?!
}

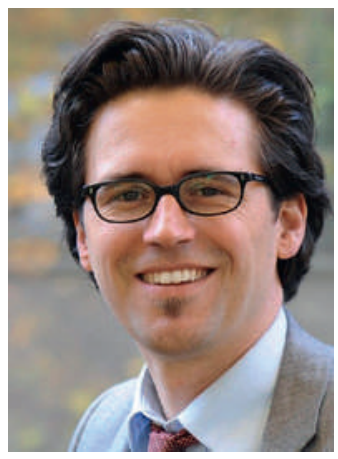

Rouven Porz
La conférence d'éthique médicale EACME (European Association of Centres of Medical Ethics) de cette année a eu lieu du 2 au 4 octobre à Lille, en France. Le thème de la conférence était le suivant: fragilité, vulnérabilité et participation sociale (fragility, vulnerability and social participation) [1]. La conférence abordait ainsi les thèmes situés dans la zone de tension entre ce qui est éthiquement possible et ce qui s'impose dans le traitement du handicap, du vieillissement de la société, des maladies psychiatriques, etc.

Personnellement, je trouvais (et je trouve toujours) qu'il était vraiment important qu'une conférence éthique étudie ces sujets d'un œil nouveau. Et qu'elle renonce un peu à la perspective de l'individu autonome et capable d'autodétermination, qu'elle adopte pour une fois consciemment celle des relations sociales, du vivre ensemble, et qu'elle fasse le lien entre ces circonstances sociales et les membres de la société qui n'ont pas toujours si facilement la possibilité d'être autonomes et de s'autodéterminer, comme l'idéal de l'autodétermination voudrait nous le faire croire. La vulnérabilité serait alors une correction raisonnée de la capacité d'autonomie. Je me suis déjà souvent interrogé sur le fait que le concept de l'autonomie constitue dans l'éthique occidentale un idéal indéfini, et rarement remis en question. sait ce qu'il dit lorsqu'il utilise le terme «vulnérable». Mais je n'en suis pas certain. Barry Hoffmaster, professeur de philosophie participant activement à la réflexion dans le domaine de l'éthique médicale, est du même avis. Il écrit non sans ironie: Vulnerability is one of those general notions we bandy about confidently but carelessly, assuming that we know what it means and that it means the same thing for everybody [2]. Mais manifestement, la vulnérabilité n'a pas la même signification pour tout le monde. Et l'on trouve effectivement des approches très distinctes de compréhension du terme. Personnellement, lorsque j'ai souhaité me faire une idée personnelle plus claire de la manière dont on peut l'interpréter, je suis tombé sur au moins quatre possibilités [3]. 1. La vulnérabilité peut être comprise du point de vue biologique, comme une soumission à la nature: aux catastrophes, aux maladies, etc. 2. Elle peut aussi être comprise d'un point de vue sociologique, comme une composante à prendre en considération dans le cadre des processus sociaux entre individus. 3 . La vulnérabilité peut s'interpréter d'un point de vue philosophique comme élément de la condition humaine, comme caractéristique existentielle. 4. Elle peut porter aussi sur un aspect plutôt médical relatif à des individus concrets, et exprimer la possibilité

\section{«Tout à coup, chacun devient hautement vulnérable!»}

\footnotetext{
* Le Dr phil., dipl. biol. Rouven Porz dirige le Service d'éthique clinique de l'Inselspital/Spital Netz Bern AG (Berne); il est également convié en tant que scientifique de l'éthique médicale à Zurich et Amsterdam, secrétaire général de l'European Association of Centres of Medical Ethics (EACME) et membre de la Rédaction Ethique du BMS.
}

Malheureusement, j'ai pu constater également à Lille que la même imprécision semble apparaître désormais dans le concept de vulnérabilité. Ou, pour l'exprimer plus simplement: Tout à coup, chacun devient hautement vulnérable! Bien sûr, une femme enceinte est vulnérable, une personne handicapée est vulnérable, les participantes et participants à des projets de recherche sont vulnérables. Mais qu'est-ce que cela veut dire précisément? Et quelqu'un s'est-il déjà donné la peine de demander à ces personnes si elles se sentaient réellement «vulnérables»? Ou la vulnérabilité devient-elle désormais un moyen de décrire (déprécier!) les personnes que nous considérons comme particulière nécessiteuses et dignes de protection? C'est possible, mais prudence: car il s'agit déjà presque de discrimination!

«Tout à coup, tout un chacun devient hautement vulnérable!»

D'accord, peut-être que je ne devrais pas m'énerver sur des concepts confus, peut-être que chacun d'une blessure psychique ou physique pouvant leur être infligée par d'autres personnes ou par des événements, et dans quelle mesure. A mon avis, ces quatre approches présentent un être humain relativement passif. L'intérêt est porté non pas sur les possibilités d'action de l'individu, mais plutôt sur la manière dont un individu apparemment passif peut être traité par l'environnement ou par d'autres personnes. Et c'est précisément cette passivité pure de l'individu qui me semble présenter des dangers en termes conceptuels, car elle implique un léger risque de discrimination.

Rouven Porz *

\section{Cf. www.eacmeweb.com}

2 Hoffmaster B. What does vulnerability mean? Dans: Hastings Center Report. Mars-avril 2006. p. 38.

3 Porz R. Zwischen Entscheidung und Entfremdung. Münster: Mentis; 2008. p. 283. 